\title{
REFLECTION AND TRANSMISSION OF PHASE-INVERSION-WAVES IN OSCILLATORS COUPLED BY TWO KINDS INDUCTORS
}

\author{
Masayuki YAMAUCHI $\dagger$, Yoshifumi NISHIO $\dagger, \ddagger$ and Akio USHIDA $\dagger$ \\ $\dagger$ Dept. of Electrical and Electronic Engineering, Tokushima University, Japan \\ $\ddagger$ Dept. of Communication Systems, Swiss Federal Institute of Technology Lausanne, Switzerland \\ \{masa,nishio,ushida\}@ee.tokushima-u.ac.jp
}

\begin{abstract}
In this study, nonlinear wave phenomena related to reflections and transmissions of the phase-inversion-waves around the discontinuity of the system consisting of two parts of coupled van der Pol oscillators are investigated. Behavior of the phase-inversion-waves around a discontinuity in a coupled system consisting of two kinds of arrays of van der Pol oscillators classified into 8 types by computer simulations. Further, the mechanisms of the reflection of the phaseinversion-waves at the discontinuity are explained.
\end{abstract}

\section{INTRODUCTION}

Large number of coupled limit-cycle oscillators are useful as models for a wide variety of systems in natural fields, for example, diverse physiological organs including gastrointestinal tracts and axial fiber of nervous systems, convecting fluids, arrays of Josephson junctions and so on. Hence, it is very important to analyze synchronization and the related phenomena observed in coupled oscillators in order to clarify mechanisms of generations or in order to control the generating-conditions of various phenomena in such natural systems. In the field of the electrical engineering, a lot of studies on synchronization phenomena of coupled van der Pol oscillators have been carried out up to now ([1]-[5] and references therein). Recently, we have discovered very interesting wave propagation phenomena of phase states between two adjacent van der Pol oscillators coupled by inductors as a ladder [6]. In the study, we named the continuously existing wave of changing phase states between two adjacent oscillators from in-phase to anti-phase or from antiphase to in-phase as "phase-inversion-wave." Further, we reported the detail on "phase-wave" in [7], which is propagation of the phase difference less than 180 degrees between two adjacent oscillators. The phase-waves exist only in the transient states unlike the phase-inversion-waves.

In this study, we observe behavior of the phase-inversionwaves around a discontinuity in a coupled system consisting of two kinds of arrays of van der Pol oscillators, namely van der Pol oscillators coupled by inductors $L_{00}$ and those coupled by $L_{01}$. Further, we explain the mechanisms of the reflection of the phase-inversion-waves at the discontinuity.

\section{CIRCUIT MODEL}

Circuit model is shown in Fig. 1. From the 1st to the $N_{1}$ th oscillators they are coupled by inductors $L_{00}$, while from the $N_{1}$ th to the $N_{2}$ th oscillators they are coupled by $L_{01}$. Namely, in this model there is a discontinuity at the $N_{1}$ th oscillator. At first, the $v-i$ characteristics of nonlinear negative resistors in the circuit are assumed by the following functions.

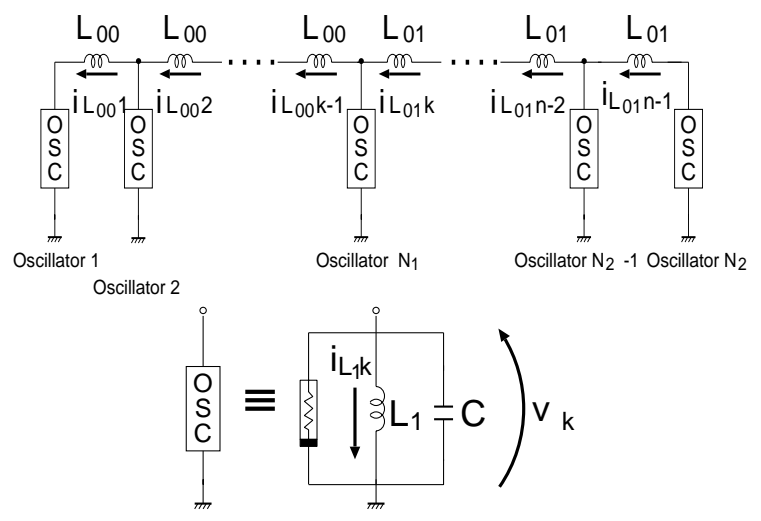

Figure 1: Circuit Model.

$$
i_{T}\left(v_{k}\right)=-g_{1} v_{k}+g_{3} v_{k}^{3} \quad\left(g_{1}, g_{3}>0\right)
$$

The circuit equations governing the circuit in Fig. 1 are expressed as

[1st oscillator]

$$
\begin{aligned}
& \dot{x}_{1}=y_{1} \\
& \dot{y}_{1}=-x_{1}+\alpha_{0}\left(x_{2}-x_{1}\right)+f\left(y_{1}\right) \\
& \left(N_{1}-1\right) \text { th oscillators] } \\
& \dot{x}_{k}=y_{k} \\
& \dot{y}_{k}=-x_{k}+\alpha_{0}\left(x_{k+1}-2 x_{k}+x_{k-1}\right)+f\left(y_{k}\right)
\end{aligned}
$$$$
\text { [2nd } \sim\left(N_{1}-1\right) \text { th oscillators] }
$$

[N $N_{1}$ th oscillator $]$

$$
\begin{aligned}
& \dot{x}_{N_{1}}=y_{N_{1}} \\
& \dot{y}_{N_{1}}=-x_{N_{1}} \\
& \quad+\alpha_{0}\left(x_{N_{1}+1}-x_{N_{1}}\right) \\
& \\
& \quad+\alpha_{1}\left(x_{N_{1}-1}-x_{N_{1}}\right)+f\left(y_{N_{1}}\right)
\end{aligned}
$$

$\left[\left(N_{1}+1\right)\right.$ th $\sim\left(N_{2}-1\right)$ th oscillators $]$

$$
\begin{aligned}
& \dot{x}_{k}=y_{k} \\
& \dot{y}_{k}=-x_{k}+\alpha_{1}\left(x_{k+1}-2 x_{k}+x_{k-1}\right)+f\left(y_{k}\right)
\end{aligned}
$$

[ $N_{2}$ th oscillator $]$

$$
\begin{aligned}
& \dot{x}_{N_{2}}=y_{N_{2}} \\
& \dot{y}_{N_{2}}=-x_{N_{2}}+\alpha_{1}\left(x_{N_{2}-1}-x_{N_{2}}\right)+f\left(y_{N_{2}}\right)
\end{aligned}
$$

where

$$
f\left(y_{k}\right)=\varepsilon\left(y_{k}-\frac{1}{3} y_{k}^{3}\right)
$$

and

$$
\begin{aligned}
& t=\sqrt{L_{1} C} \tau, i_{L_{1} k}=\sqrt{\frac{C g_{1}}{3 L_{1} g_{3}}} x_{k}, v_{k}=\sqrt{\frac{g_{1}}{3 g_{3}}} y_{k}, \\
& \alpha_{0}=\frac{L_{1}}{L_{00}}, \alpha_{1}=\frac{L_{1}}{L_{01}}, \varepsilon=g_{1} \sqrt{\frac{L_{1}}{C}}, \frac{d}{d \tau}=" . " \text {.(8) }
\end{aligned}
$$


It should be noted that $\alpha$ corresponds to the coupling and that $\varepsilon$ corresponds to the nonlinearity. Equations (2)(8) are calculated for the cases of $\left(N_{1}, N_{2}\right)=(9,17)$ and $(21,30)$ by using the fourth-order Runge-Kutta method.

\section{REFLECTION AND TRANSMISSION}

In this section we observe reflection and transmission of the phase-inversion-waves for the case of $\left(N_{1}, N_{2}\right)=(9,17)$. We fix the parameters $\varepsilon=0.20$ and $\alpha_{0}=0.050$ and $\alpha_{1}$ is varied as a control parameter. In the computer calculations, we produced phase-inversion-waves as follows. Almost same initial conditions are given for all oscillators to produce complete in-phase synchronization, which is one stable steady states in the system. Next, invert the voltage and the current of the first oscillator.

Fig. 2 shows the observed result. In diagrams, vertical axes are sum of voltages of adjacent oscillators and horizontal axes are time. The observed phenomena are classified into eight types as follows.

(a) around 0.001: The phase-inversion-waves are reflected at the discontinuity almost completely.

(b) around 0.002 0.018: The phase-inversion-waves are reflected and transmitted. But, the phase-inversionwaves change to the phase-waves after the transmission.

(c) around 0.019 0.025: The phase-inversion-waves are reflected and transmitted and do not change to the phase-waves after the transmission.

(d) around 0.026 0.043: The phase-inversion-waves are reflected and transmitted. But, the phase-inversionwaves change to the phase-waves after the reflection.

(e) around 0.044 0.056: The phase-inversion-waves are transmitted almost completely. The phase-inversionwaves transmitted completely when $\alpha_{1}$ is equal to 0.050 .

(f) around 0.057 0.130: The phase-inversion-waves are transmitted. The phase-waves are generated after the phase-inversion-waves are transmitted.

(g) around 0.131 0.173: The phase-inversion-waves are reflected and transmitted. But, the phase-inversionwaves change to the phase-waves after the reflection and transmission.

(h) around $0.174 \sim$ : The phase-inversion-waves are reflected and transmitted. But, the phase-inversionwaves change to the phase-waves after the transmission.

It should be noted that we could observe different transmission phenomena for the cases of $L_{01}>>L_{00}$ and $L_{01}<<$ $L_{00}$. Namely, for $L_{01}>>L_{00}\left(\alpha_{1}<<0.05\right)$ phase-inversionwave is not transmitted beyond the discontinuity. While, for $L_{01}<<L_{00}\left(\alpha_{1}>>0.05\right)$ phase-inversion-wave is transmitted as phase-wave beyond the discontinuity. This interesting difference will be explained in the Sec. 5 .

Figure 3 summarizes the above bifurcation.

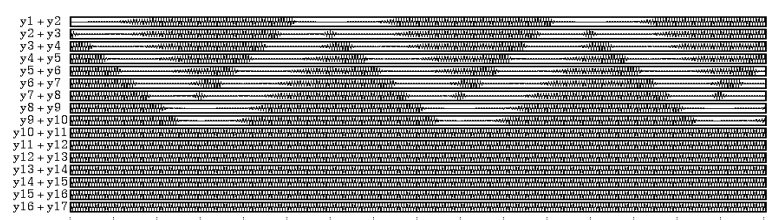

(a) $\alpha_{1}=0.0001$.

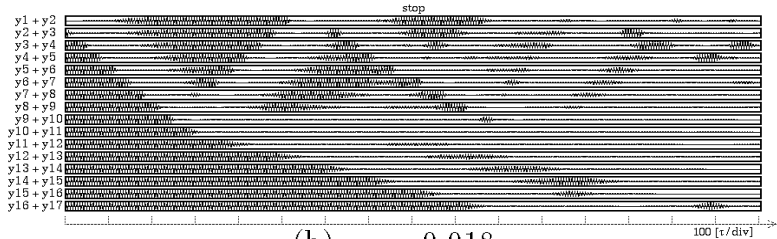

(b) $\alpha_{1}=0.018$.

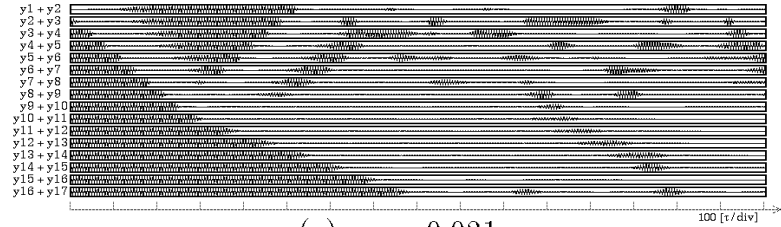

(c) $\alpha_{1}=0.021$.

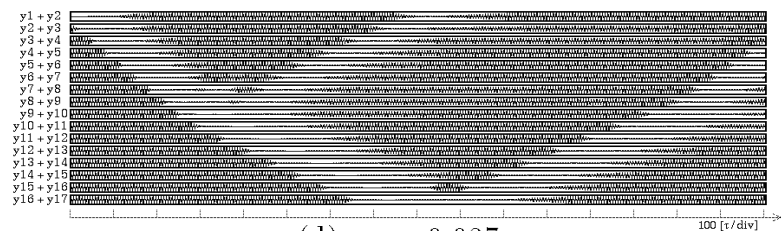

(d) $\alpha_{1}=0.027$.

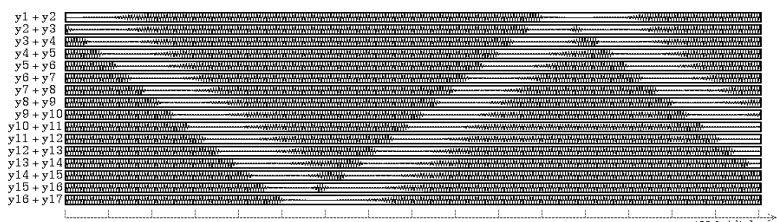

(e) $\alpha_{1}=0.046$

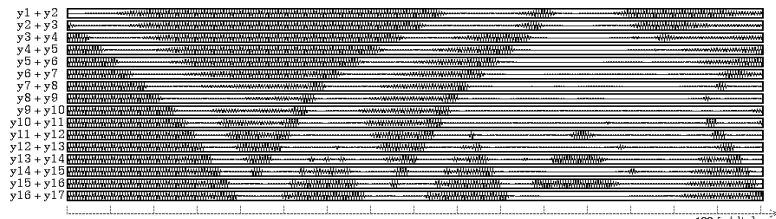

(f) $\alpha_{1}=0.100$

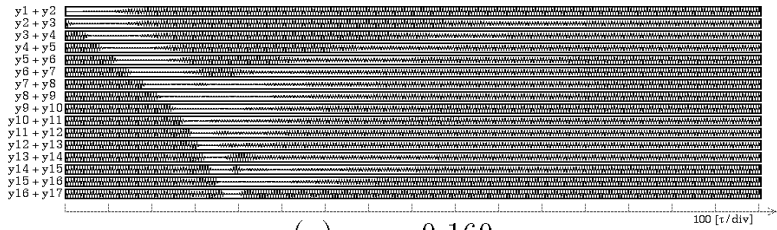

(g) $\alpha_{1}=0.160$

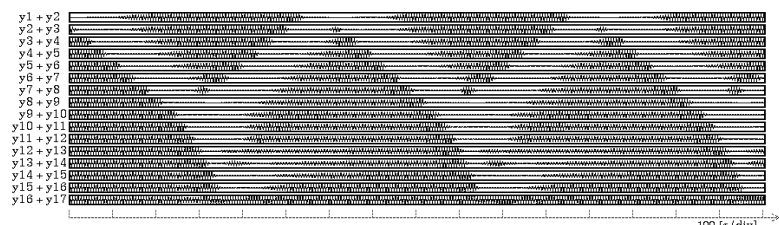

(h) $\alpha_{1}=0.240$.

Figure 2: Observation of the reflection and the transmission of the phase-inversion-waves. $N_{1}=9, N_{2}=17$, $\alpha_{0}=0.050$ and $\varepsilon=0.20$. 


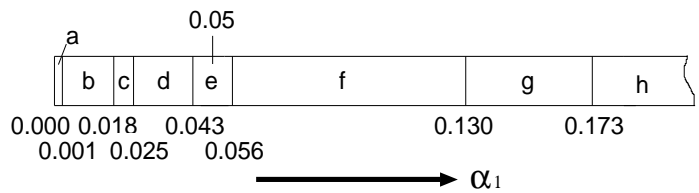

Figure 3: Bifurcation diagram. $N_{1}=9, N_{2}=17, \alpha_{0}=$ 0.050 and $\varepsilon=0.20$.

\section{COMPARISON WITH WAVES IN TRANSMISSION LINE}

The reflection and transmission observed in our system seem to be similar to wave phenomena observed in two transmission lines with different characteristic impedances joined in tandem. However, we found two major different phenomena in our system as follows:

1. After the reflection or the transmission, the phaseinversion-waves often changes their characteristics, namely we can observe that the phase-inversion-waves change to phase-waves with phase difference less than 180 degrees for wide range of control parameter value.

2. The reflection or the transmission may occurs two times when the phase-inversion-waves arrives at the discontinuity. The reason of this phenomena is could be explained as follows. The oscillator at the discontinuity needs longer time to returns to in-phase synchronization frequency than time the other oscillators need, after the phase-inversion-waves arrived at the oscillator. This produces new phase difference between the oscillator at the discontinuity and its adjacent oscillators and it results the generation of the second phase-wave.

\section{MECHANISM OF REFLECTION}

In this section, the reflection mechanism of the phaseinversion-waves at the discontinuity is explained according to the computer calculated results for the case of $\left(N_{1}, N_{2}\right)=$ $(21,30)$.

It has been already known that oscillation frequency of in-phase synchronization of oscillators coupled by inductors is different from that of anti-phase synchronization. Namely, $f_{i n}$, oscillation frequency of in-phase synchronization, is smaller than $f_{a n t i}$, oscillation frequency of antiphase synchronization. Further, the difference between $f_{i n}$ and $f_{a n t i}$ increases as coupling inductance increases. We use this characteristic to explain the mechanism of the reflection.

5.1 When $L_{01}>>L_{00}$ (i.e. $\alpha_{1}<<\alpha_{0}$ ).

1. Let us assume that OSC20 OSC30 are in-phase synchronization and that a phase-inversion-wave from in-phase to anti-phase reaches at OSC20 from the direction of OSC1.
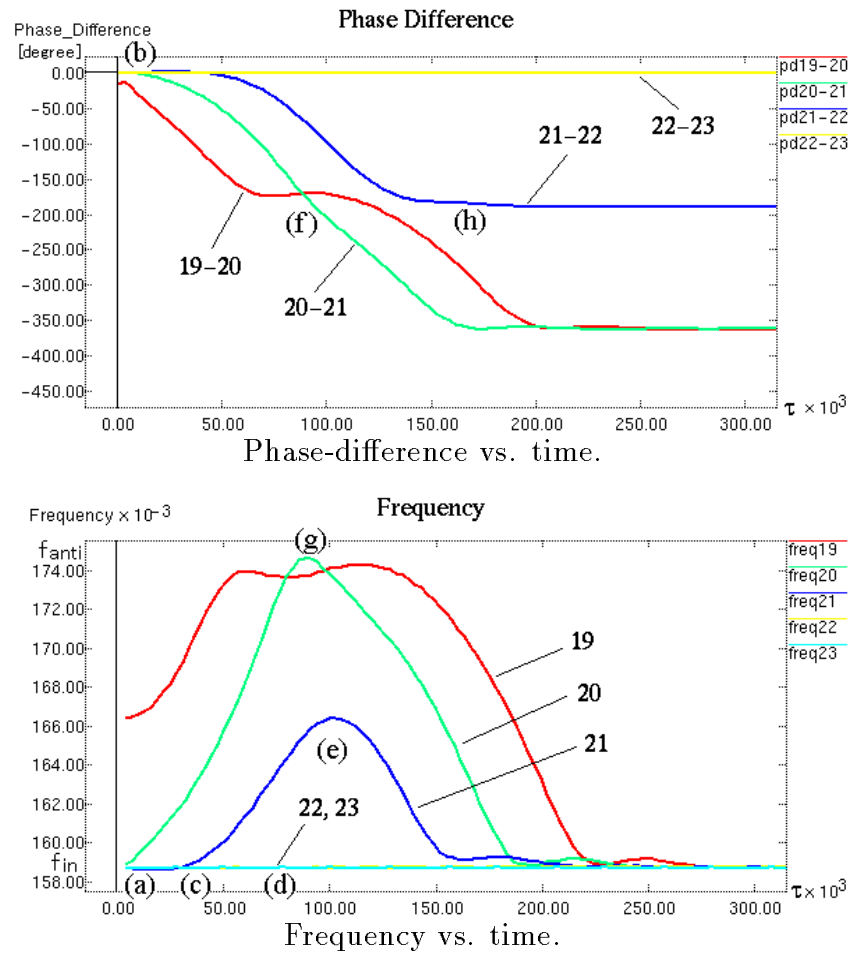

Figure 4: Reflection at the discontinuity for the case of $\alpha_{1} \ll<\alpha_{0} . N_{1}=21, N_{2}=30, \alpha_{0}=0.0500, \alpha_{1}=0.0001$ and $\varepsilon=0.20$.

2. Oscillation frequency of OSC20 $f_{20}$ begins to change from $f_{i n}$ toward $f_{\text {anti }}$, because in-phase synchronization between OSC19 and OSC20 breaks by the phaseinversion-wave (see (a) in Fig.4).

3. As oscillation frequency of OSC20 $f_{20}$ changes from $f_{i n}$ to $f_{\text {anti }}$, phase difference between OSC20 and OSC21 $\Phi_{20,21}$ approaches $-\pi$ (see (b) in Fig.4).

4. As $\Phi_{20,21}$ approaches $-\pi$, oscillation frequency of OSC21 $f_{21}$ begins to change from $f_{i n}$ to $f_{\text {anti }}$ (see (c) in Fig.4).

5. Change of $f_{21}$ does not propagate to OSC22 immediately, because $L_{01}$ is much larger than $L_{00}$ (see (d) in Fig.4).

6. Hence, $f_{21}$ cannot reach $f_{a n t i}$ and begins returning to $f_{i n}$ (see (e) in Fig.4).

7. As $f_{21}$ returns to $f_{i n}, \Phi_{20,21}$ continues to increase until reaching $-2 \pi$ (see (f) in Fig.4).

8. By the effect of the decrease of $\Phi_{20,21}, f_{20}$ begins to decreases again from $f_{a n t i}$ toward $f_{i n}$ (see $(\mathrm{g})$ in Fig.4).

9. $\Phi_{21,22}$ becomes $\pi$, because in spite of constant value which is $f_{22}, f_{21}$ changes from $f_{i n}$ and returns to $f_{i n}$ again (see (h) in Fig.4). 
As $L_{01}$ approaches $L_{00}$, it becomes easier that change of $f_{21}$ propagates to OSC22. Hence, we could observe that the phase-inversion-wave is transmitted beyond the discontinuity as shown in Figs. 3(b)(c)(d). While, the reflection caused by the fact that the change of $f_{21}$ is hard to propagate becomes smaller.

5.2 When $L_{01}<<L_{00}$ (i.e. $\alpha_{1}>>\alpha_{0}$ ).
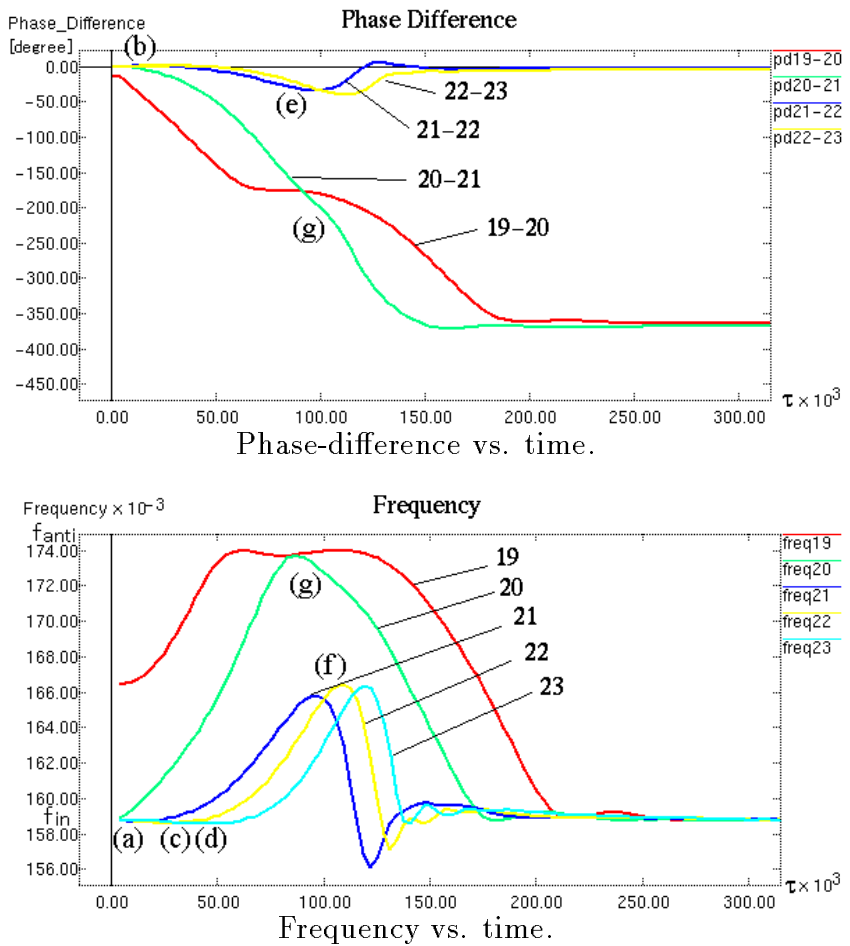

Figure 5: Reflection at the discontinuity for the case of $\alpha_{1}>>\alpha_{0} . N_{1}=21, N_{2}=30, \alpha_{0}=0.00500, \alpha_{1}=0.20000$ and $\varepsilon=0.20$.

1. Let us assume that OSC20 OSC30 are in-phase synchronization and that a wave from in-phase to antiphase reaches at OSC20 from the direction of OSC1.

2. Oscillation frequency of OSC20 $f_{20}$ begins to change from $f_{i n}$ toward $f_{\text {anti }}$, because in-phase synchronization between OSC19 and OSC20 breaks by the phaseinversion-wave (see (a) in Fig.5).

3. As oscillation frequency of OSC20 $f_{20}$ changes from $f_{i n}$ to $f_{\text {anti }} \Phi_{20,21}$ approaches $-\pi$ (see (b) in Fig.5).

4. As $\Phi_{20,21}$ approaches $-\pi$, oscillation frequency of OSC21 $f_{21}$ changes from $f_{i n}$ to $f_{a n t i}$ (see (c) in Fig.5).

5. Change of $f_{21}$ propagates to OSC22 immediately, because $L_{01}$ is much smaller than $L_{00}$ (see (d) in Fig.4).

6. Hence, in-phase synchronization between OSC21 and OSC22 is hard to break. (see (e) in Fig.5). And $f_{21}$ and $f_{22}$ return to $f_{i n}$ together (see (f) in Fig.5).

7. As $f_{21}$ returns to $f_{i n}, \Phi_{20,21}$ continues to increase until reaching $-2 \pi$ (see (g) in Fig.5).
Hence, when $L_{01}$ is much smaller than $L_{00}$, the transmission waves always exist.

As $L_{01}$ approaches $L_{00}$, it becomes more difficult that phase states of OSC21 and OSC22 hold in-phase synchronization. Hence, the reflection of the phase-inversion-waves is disappear as shown in Figs. $3(\mathrm{f})(\mathrm{g})$.

\section{CONCLUSIONS}

In this study, we observed behavior of the phase-inversionwaves around the discontinuity of the system consisting of two parts of coupled van der Pol oscillators. By computer calculations observed phenomena were classified into 8 types. Further, we could explain the mechanism of the reflection of the phase-inversion-wave at the discontinuity and made clear that different mechanisms reflect the waves fro the cases of $L_{01}>L_{00}$ and $L_{01}<L_{00}$.

\section{REFERENCES}

[1] D. A. Linkens "Analytical Solution of Large Numbers of Mutually Coupled Nearly Sinusoidal Oscillators" IEEE Trans. Circuits Syst., vol. 21, no. 2, pp. 294-300, Mar. 1974.

[2] T. Endo and S. Mori "Mode Analysis of a Multimode Ladder Oscillator" IEEE Trans. Circuits Syst., vol. 23, no. 2, pp. 100-113, Feb. 1976.

[3] T. Endo and S. Mori "Mode Analysis of TwoDimensional Low-Pass Multimode Oscillator" IEEE Trans. Circuits Syst., vol. 23, no. 9, pp. 517-530, Sep. 1976.

[4] T. Endo and S. Mori "Mode Analysis of a Ring of a Large Number of Mutually Coupled van der Pol Oscillators" IEEE Trans. Circuits Syst., vol. 25, no. 1, pp. 7-18, Jan. 1978.

[5] S. Moro, Y. Nishio and S. Mori "Synchronization Phenomena in Oscillators Coupled by One Resistor" IEICE Trans. Fundamentals, vol. E78-A, no. 2, pp. 244253, Feb. 1995.

[6] M. Yamauchi, M. Wada, Y. Nishio and A. Ushida "Wave Propagation Phenomena of Phase States in Oscillators Coupled by Inductors as a Ladder" IEICE Trans. Fundamentals, vol. E82-A, no. 11, pp. 25922598, Nov. 1999.

[7] M. Yamauchi, M. Wada, Y. Nishio and A. Ushida "Analysis of Phase Difference Propagation in Oscillators Coupled by Inductors as a Ladder" Proc. NOLTA'99, vol. 2, pp. 783-786, Nov. 1999. 